Article

\title{
Iterative Algorithms for a System of Variational Inclusions in Banach Spaces
}

\author{
Lu-Chuan Ceng ${ }^{1}\left(\mathbb{D}\right.$, Mihai Postolache $2,3,4, * \mathbb{( D )}$ and Yonghong Yao ${ }^{5,6}$
}

1 Department of Mathematics, Shanghai Normal University, Shanghai 200234, China; zenglc@hotmail.com

2 Center for General Education, China Medical University, Taichung 40402, Taiwan

3 Romanian Academy, Gh. Mihoc-C. Iacob Institute of Mathematical Statistics and Applied Mathematics, 050711 Bucharest, Romania

4 University "Politehnica" of Bucharest, Department of Mathematics and Informatics, 060042 Bucharest, Romania

5 School of Mathematical Sciences, Tianjin Polytechnic University, Tianjin 300387, China; yaoyonghong@aliyun.com

6 The Key Laboratory of Intelligent Information and Data Processing of NingXia Province, North Minzu University, Yinchuan 750021, China

* Correspondence: emscolar@yahoo.com

Received: 26 May 2019; Accepted: 18 June 2019; Published: 19 June 2019

Abstract: A system of variational inclusions (GSVI) is considered in Banach spaces. An implicit iterative procedure is proposed for solving the GSVI. Strong convergence of the proposed algorithm is given.

Keywords: system of variational inclusions; accretive mapping; strict pseudocontraction; implicit iterative procedure

MSC: 47H05; 47H10; 47J25

\section{Introduction}

Let $X$ be a smooth Banach space and $\varnothing \neq C \subset X$ a closed convex set. Let $A_{1}, A_{2}: C \rightarrow X$ and $M_{1}, M_{2}: C \rightarrow 2^{X}$ be nonlinear mappings. In the present article, we consider the following system of variational inclusions (GSVI, for short) which aims to seek $\left(u^{*}, v^{*}\right) \in C \times C$ verifying

$$
\left\{\begin{array}{l}
0 \in \varsigma_{1}\left(A_{1} v^{*}+M_{1} u^{*}\right)+u^{*}-v^{*}, \\
0 \in \varsigma_{2}\left(A_{2} u^{*}+M_{2} v^{*}\right)+v^{*}-u^{*},
\end{array}\right.
$$

where $\varsigma_{1}$ and $\varsigma_{2}$ are two positive constants.

Special cases: If $A_{1}=A_{2}=A$ and $M_{1}=M_{2}=M$, then the relation (1) reduces to seek $\left(u^{*}, v^{*}\right) \in C \times C$ verifying

$$
\left\{\begin{array}{l}
0 \in \varsigma_{1}\left(A v^{*}+M u^{*}\right)+u^{*}-v^{*}, \\
0 \in \varsigma_{2}\left(A u^{*}+M v^{*}\right)+v^{*}-u^{*} .
\end{array}\right.
$$

If $u^{*}=v^{*}$ in (2), then the relation (2) reduces to seek $\left(u^{*}, v^{*}\right) \in C \times C$ verifying

$$
0 \in A u^{*}+M u^{*} .
$$


Especially, if $M=\partial \phi$, where $\phi: H \rightarrow R \cup+\infty$ is a proper convex lower semi-continuous function, then we have the following mixed quasi-variational inequality

$$
\langle A u, y-u\rangle+\phi(y)-\phi(u)\rangle \geq 0, \forall y \in H .
$$

Variational inequalities and variational inclusions have played vital roles in practical applications. Numerous iterative procedures for approaching variational inequalities and variational inclusions have been computed by the researchers [1-29].

In [4], the authors introduced an iterative procedure for approaching GSVI (1). Qin et al. [30] suggested an extragradient algorithm for solving GSVI (1), and demonstrated the strong convergence analysis of the presented algorithm. Lan et al. [28], Buong et al. [11], Zhang et al. [13] studied iterative procedures for approaching variational inclusion (3).

On the other hand, iterative computation of zeros or fixed points of nonlinear operators has been studied extensively in the literature [14,31-36]. Zhang et al. [37] introduced an iterative procedure for approaching a solution of the inclusion problem (3) and a fixed point of a nonexpansive mapping in Hilbert spaces. Peng et al. [38] presented a viscosity algorithm for finding a solution of a variational inclusion with set-valued maximal monotone mapping and inverse strongly monotone mappings, the set of solutions of an equilibrium problem and a fixed point of a nonexpansive mapping.

Motivated by the above work, in the present paper, we consider the GSVI (1) with the hierarchical variational inequality constraint for a strict pseudocontraction $T$ in Banach spaces. We suggest an implicit iterative procedure for solving the GSVI (1) with the HVI constraint for strict pseudocontraction $T$. We show the strong convergence of the suggested procedure to a solution of the GSVI (1).

\section{Preliminaries}

Let $X$ be a real Banach space and $\varnothing \neq C \subset X$ a closed convex set. A mapping $f: C \rightarrow C$ is said to be $k$-Lipschitz if $\|f(u)-f(v)\| \leq k\|u-v\|, \forall u, v \in C$ for some $k \geq 0$. If $k<1$, then $f$ is said to be a $k$-contraction. If $k=1$, then $f$ is said to be nonexpansive.

Recall that an operator $T: C \rightarrow X$ is called

(i) accretive if

$$
\langle T u-T v, j(u-v)\rangle \geq 0, \forall u, v \in C,
$$

where $j(u-v) \in J(u-v)$.

(ii) $\alpha$-inverse-strongly accretive if

$$
\langle T u-T v, j(u-v)\rangle \geq \alpha\|T u-T v\|^{2}, \forall u, v \in C,
$$

where $j(u-v) \in J(u-v)$ and $\alpha>0$.

(iii) strictly pseudocontractive if

$$
\langle T u-T v, j(u-v)\rangle \leq\|u-v\|^{2}-\beta\|u-v-(T u-T v)\|^{2}, \forall u, v \in C,
$$

where $j(u-v) \in J(u-v)$ and $\beta>0$.

If $X$ is $q$-uniformly smooth with $1<q \leq 2$, then

$$
\|u+v\|^{q}+\|u-v\|^{q} \leq 2\left(\|u\|^{q}+\|c v\|^{q}\right), \forall u, v \in X,
$$

where $c>0$ is some constant.

Proposition 1 ([32]). In a smooth and uniformly convex Banach space $X$, for all $u, v \in B_{r}=\{u \in X:\|u\| \leq$ $r\}$, there holds

$$
g(\|u-v\|) \leq\|u\|^{2}-2\langle u, j(v)\rangle+\|v\|^{2},
$$


where $g:[0,2 r] \rightarrow \mathbf{R}$ is a strictly increasing, continuous, and convex function satisfying $g(0)=0$.

Proposition 2 ([35]). In a 2-uniformly smooth Banach space X, there holds

$$
\|u+v\|^{2} \leq\|u\|^{2}+2\langle v, j(u)\rangle+2\|c v\|^{2}, \forall u, v \in X .
$$

Let $D \subset C$ and $\Pi: C \rightarrow D$ be an operator. If $\Pi[(1-s) \Pi(u)+s u]=\Pi(u)$, whenever $(1-$ $s) \Pi(u)+s u \in C$ for $u \in C$ and $s \geq 0$, we call $\Pi$ is sunny.

Proposition 3 ([26]). Let $X$ be a smooth Banach space and $\varnothing \neq C \subset X$ a closed convex set. Let $\varnothing \neq D \subset C$ be a set and $\Pi: C \rightarrow D$ be a retraction. Then the following conclusions are equivalent:

(i) $\langle\Pi(u)-u, j(v-\Pi(u))\rangle \geq 0, \forall u \in C$ and $\forall v \in D$;

(ii) $\|\Pi(u)-\Pi(v)\|^{2} \leq\langle u-v, j(\Pi(u)-\Pi(v))\rangle, \forall u, v \in C$;

(iii) $\Pi$ is sunny nonexpansive operator.

If an accretive operator $M$ satisfies $R(I+r M)=X$ for each $r>0$, then $M$ is said to be $m$-accretive. Assume that an accretive $M$ satisfies the range condition $\overline{D(M)} \subset R(I+r M)$. Define the resolvent $J_{r}^{M}: R(I+r M) \rightarrow D(M)$ of $M$ by $J_{r}^{M}=(I+r M)^{-1}$. Note that $J_{r}^{M}$ is nonexpansive and $F\left(J_{r}^{M}\right)=$ $M^{-1} 0=\{x \in D(M): 0 \in M x\}$ [31]. If $M^{-1} 0 \neq \varnothing$, then the inclusion $0 \in M x$ is solvable.

Lemma 1. Let $X$ be a smooth Banach space and $\varnothing \neq C \subset X$ a closed convex set. Let $M: C \rightarrow 2^{X}$ be an $m$-accretive operator. Then, for any given $r>0$,

$$
\left\|J_{r}^{M} x-J_{r}^{M} y\right\|^{2} \leq\left\langle x-y, j\left(J_{r}^{M} x-J_{r}^{M} y\right)\right\rangle, \forall x, y \in X .
$$

This means that $J_{r}^{M}: X \rightarrow C$ is nonexpansive.

Proof. Put $u=J_{r}^{M} x$ and $v=J_{r}^{M} y$. Then we have $x \in(I+r M) u$ and $y \in(I+r M) v$. Hence, there exist $\tilde{u} \in M u$ and $\tilde{v} \in M v$ such that $x=u+r \tilde{u}$ and $y=v+r \tilde{v}$. Utilizing the accretiveness of $M$, we obtain

$$
\begin{aligned}
\left\langle x-y, j\left(J_{r}^{M} x-J_{r}^{M} y\right)\right\rangle & =\langle u+r \tilde{u}-(v+r \tilde{v}), j(u-v)\rangle \\
& =\langle u-v, j(u-v)\rangle+r\langle\tilde{u}-\tilde{v}, j(u-v)\rangle \\
& =\|u-v\|^{2}+r\langle\tilde{u}-\tilde{v}, j(u-v)\rangle \\
& \geq\|u-v\|^{2} \\
& =\left\|J_{r}^{M} x-J_{r}^{M} y\right\|^{2} .
\end{aligned}
$$

Lemma 2. Let $M_{1}, M_{2}: C \rightarrow 2^{X}$ be two m-accretive operators and $A_{1}, A_{2}: C \rightarrow X$ be two operators. $\left(x^{*}, y^{*}\right)$ is a solution of the GSVI (1) iff $Q x^{*}=J_{\varsigma_{1}}^{M_{1}}\left(I-\varsigma_{1} A_{1}\right) J_{\zeta_{2}}^{M_{2}}\left(I-\varsigma_{2} A_{2}\right) x^{*}$, where $y^{*}=J_{\varsigma_{2}}^{M_{2}}\left(I-\varsigma_{2} A_{2}\right) x^{*}$.

Proof. Observe that

$$
\left\{\begin{array} { l } 
{ 0 \in x ^ { * } - y ^ { * } + \varsigma _ { 1 } ( A _ { 1 } y ^ { * } + M _ { 1 } x ^ { * } ) } \\
{ 0 \in y ^ { * } - x ^ { * } + \varsigma _ { 2 } ( A _ { 2 } x ^ { * } + M _ { 2 } y ^ { * } ) }
\end{array} \Leftrightarrow \left\{\begin{array}{l}
x^{*}=J_{\varsigma_{1}}^{M_{1}}\left(I-\varsigma_{1} A_{1}\right) y^{*}, \\
y^{*}=J_{\varsigma_{2}}^{M_{2}}\left(I-\varsigma_{2} A_{2}\right) x^{*}
\end{array} \Leftrightarrow x^{*}=Q x^{*} .\right.\right.
$$

Lemma 3 ([3]). Let $X$ be a strictly convex Banach space and $\varnothing \neq C \subset X$ a closed convex set. Let $\mu \in(0,1)$ be a constant. Define an operator $S: C \rightarrow X$ by $S x=\mu T_{1} x+(1-\mu) T_{2} x, \forall x \in C$, where $T_{1}, T_{2}: C \rightarrow X$ be two nonexpansive mappings with $F\left(T_{1}\right) \cap F\left(T_{2}\right) \neq \varnothing$. Then $S$ is nonexpansive and $F(S)=F\left(T_{1}\right) \cap F\left(T_{2}\right)$. 
Lemma 4 ([3]). Let $X$ be a 2-uniformly smooth Banach space and $\varnothing \neq C \subset X$ a closed convex set. If the operator $A: C \rightarrow X$ is $\alpha$-inverse-strongly accretive, then

$$
\|(I-\zeta A) u-(I-\zeta A) v\|^{2} \leq\|u-v\|^{2}+2 \zeta\left(c^{2} \zeta-\alpha\right)\|A u-A v\|^{2}, \forall u, v \in C .
$$

Lemma 5 ([3]). Let $X$ be a 2-uniformly smooth Banach space and $\varnothing \neq C \subset X$ a closed convex set. Let $M_{1}, M_{2}$ : $C \rightarrow 2^{X}$ be two m-accretive operators and $A_{i}: C \rightarrow X(i=1,2)$ be $\zeta_{i}$-inverse-strongly accretive operator. Define an operator $Q: C \rightarrow C$ by $Q:=J_{\zeta_{1}}^{M_{1}}\left(I-\varsigma_{1} A_{1}\right) J_{\zeta_{2}}^{M_{2}}\left(I-\varsigma_{2} A_{2}\right)$. If $0 \leq \varsigma_{i} \leq \frac{\zeta_{i}}{c^{2}}(i=1,2)$, then $Q: C \rightarrow C$ is nonexpansive.

Lemma 6 ([36]). Let $X$ be a uniformly smooth Banach space and $\varnothing \neq C \subset X$ a closed convex set. Let $A$ : $C \rightarrow C$ be a nonexpansive mapping with $F(A) \neq \varnothing$, and $f: C \rightarrow X$ be a contraction. Let $t \in(0,1)$. Define a net $z_{t}$ by $z_{t}=t f\left(z_{t}\right)+(1-t) A z_{t}$. Then $z_{t} \rightarrow x^{*} \in F(A)$ and

$$
\left\langle(I-f) x^{*}, j\left(x^{*}-x\right)\right\rangle \leq 0, \quad \forall x \in F(A) .
$$

Lemma 7 ([36]). Assume the sequence $\left\{a_{n}\right\} \subset[0, \infty)$ satisfies $a_{n+1} \leq\left(1-\lambda_{n}\right) a_{n}+\lambda_{n} \sigma_{n}(\forall n \geq 0)$, where the sequences $\left\{\lambda_{n}\right\} \subset(0,1)$ and $\left\{\sigma_{n}\right\}$ satisfy

(i) $\sum_{n=0}^{\infty} \lambda_{n}=\infty$;

(ii) either $\sum_{n=0}^{\infty}\left|\lambda_{n} \sigma_{n}\right|<\infty$ or $\lim \sup _{n \rightarrow \infty} \sigma_{n} \leq 0$.

Then $\lim _{n \rightarrow \infty} a_{n}=0$.

Lemma 8 ([33]). Let $X$ be a 2-uniformly smooth Banach space and $\varnothing \neq C \subset X$ a closed convex set. Let $T$ : $C \rightarrow C$ be a $\lambda$-strict pseudocontraction. Define an operator $T_{\alpha}$ by $T_{\alpha} x=(1-\alpha) x+\alpha T x, \alpha \in(0,1)$. Then, $T_{\alpha}: C \rightarrow C$ is nonexpansive with $F\left(T_{\alpha}\right)=F(T)$ provided $\alpha \in\left(0, \frac{\lambda}{c^{2}}\right]$.

\section{Main Results}

Theorem 1. Let $X$ be a uniformly convex and 2-uniformly smooth Banach space and $\varnothing \neq C \subset X a$ closed convex set. Let $M_{1}, M_{2}: C \rightarrow 2^{X}$ be two m-accretive operators and $A_{i}: C \rightarrow X(i=1,2)$ be $\zeta_{i}$-inverse-strongly accretive operator. Let $f: C \rightarrow C$ be a contraction with coefficient $k \in[0,1)$. Let $V: C \rightarrow C$ be a nonexpansive operator and $T: C \rightarrow C$ be a $\lambda$-strict pseudocontraction with $\Omega:=F(T) \cap F(Q) \neq \varnothing$, where the operator $Q$ is defined as in Lemma 5. Assume that the sequences $\left\{\alpha_{n}\right\} \subset(0,1),\left\{\beta_{n}\right\} \subset(0,1)$, $\left\{\delta_{n}\right\} \subset(0,1)$ and $\left\{\gamma_{n}\right\} \subset(0,1)$ satisfy

(i) $\alpha_{n}+\delta_{n}+\beta_{n}+\gamma_{n}=1(\forall n \geq 1)$;

(ii) $\alpha_{n} \rightarrow 0$ and $\frac{\beta_{n}}{\alpha_{n}} \rightarrow 0$;

(iii) $\gamma_{n} \rightarrow 1$;

(iv) $\sum_{n=1}^{\infty} \alpha_{n}=\infty$.

Given $x_{0} \in C$, compute the sequences $\left\{x_{n}\right\}$ and $\left\{y_{n}\right\}$ such that

$$
\left\{\begin{array}{l}
y_{n}=J_{\varsigma_{2}}^{M_{2}}\left(x_{n}-\varsigma_{2} A_{2} x_{n}\right), \\
x_{n}=\alpha_{n} f\left(x_{n-1}\right)+\delta_{n} x_{n-1}+\beta_{n} V x_{n-1}+\gamma_{n}\left[\mu S x_{n}+(1-\mu) J_{\zeta_{1}}^{M_{1}}\left(y_{n}-\varsigma_{1} A_{1} y_{n}\right)\right], \forall n \geq 1,
\end{array}\right.
$$

where $S x=(1-\alpha) x+\alpha T x, \forall x \in C$ with $0<\alpha<\min \left\{1, \frac{\lambda}{c^{2}}\right\}$ and $\mu \in(0,1)$. Then $x_{n} \rightarrow x^{*}, y_{n} \rightarrow y^{*}$ and

(a) $\left(x^{*}, y^{*}\right)$ solves the GSVI (1);

(b) $x^{*}$ solves the variational inequality: $\left\langle(I-f) x^{*}, j\left(u-x^{*}\right)\right\rangle \geq 0, \forall u \in \Omega$. 
Proof. By Lemmas 5 and $8, Q$ and $S$ are nonexpansive and $F(S)=F(T)$. Put $A:=\mu S+(1-\mu) Q$ with $\mu \in(0,1)$. It is easy to see that the implicit iterative scheme (4) can be rewritten as

$$
x_{n}=\alpha_{n} f\left(x_{n-1}\right)+\delta_{n} x_{n-1}+\beta_{n} V x_{n-1}+\gamma_{n} A x_{n}, \forall n \geq 1 .
$$

Consider the mapping $F_{n} u=\alpha_{n} f\left(x_{n-1}\right)+\delta_{n} x_{n-1}+\beta_{n} V x_{n-1}+\gamma_{n} A u, \forall u \in C$. According to Lemma 3, we have

$$
\left\|F_{n} u-F_{n} v\right\|=\gamma_{n}\|A u-A v\| \leq \gamma_{n}\|u-v\|, \forall u, v \in C .
$$

Hence $F_{n}$ is a contraction. Thus, (5) and hence (4) are all well-posed.

Let $u^{\dagger} \in \Omega$. Thus, $T u^{\dagger}=u^{\dagger}$ and $Q u^{\dagger}=u^{\dagger}$. It is clear that

$$
\begin{aligned}
x_{n}-u^{\dagger} & =\alpha_{n} f\left(x_{n-1}\right)+\delta_{n} x_{n-1}+\beta_{n} V x_{n-1}+\gamma_{n} A x_{n}-u^{\dagger} \\
& =\alpha_{n}\left(f\left(x_{n-1}\right)-u^{\dagger}\right)+\delta_{n}\left(x_{n-1}-u^{\dagger}\right)+\beta_{n}\left(V x_{n-1}-u^{\dagger}\right)+\gamma_{n}\left(A x_{n}-u^{\dagger}\right) .
\end{aligned}
$$

By Lemma 3, we get

$$
\begin{aligned}
\left\|x_{n}-u^{\dagger}\right\| \leq & \alpha_{n}\left\|f\left(x_{n-1}\right)-u^{\dagger}\right\|+\delta_{n}\left\|x_{n-1}-u^{\dagger}\right\|+\beta_{n}\left\|V x_{n-1}-u^{\dagger}\right\|+\gamma_{n}\left\|A x_{n}-u^{\dagger}\right\| \\
\leq & \alpha_{n}\left(\left\|f\left(x_{n-1}\right)-f\left(u^{\dagger}\right)\right\|+\left\|f\left(u^{\dagger}\right)-u^{\dagger}\right\|\right)+\delta_{n}\left\|x_{n-1}-u^{\dagger}\right\| \\
& +\beta_{n}\left(\left\|V x_{n-1}-V u^{\dagger}\right\|+\left\|V u^{\dagger}-u^{\dagger}\right\|\right)+\gamma_{n}\left\|x_{n}-u^{\dagger}\right\| \\
\leq & \alpha_{n}\left(k\left\|x_{n-1}-u^{\dagger}\right\|+\left\|f\left(u^{\dagger}\right)-u^{\dagger}\right\|\right)+\delta_{n}\left\|x_{n-1}-u^{\dagger}\right\| \\
& +\beta_{n}\left(\left\|x_{n-1}-u^{\dagger}\right\|+\left\|V u^{\dagger}-u^{\dagger}\right\|\right)+\gamma_{n}\left\|x_{n}-u^{\dagger}\right\| \\
= & \alpha_{n}\left\|f\left(u^{\dagger}\right)-u^{\dagger}\right\|+\left(1-(1-k) \alpha_{n}-\gamma_{n}\right)\left\|x_{n-1}-u^{\dagger}\right\|+\beta_{n}\left\|V u^{\dagger}-u^{\dagger}\right\|+\gamma_{n}\left\|x_{n}-u^{\dagger}\right\| .
\end{aligned}
$$

By condition (ii), without loss of generality, we assume that $\beta_{n} \leq \alpha_{n}$ for all $n \geq 1$. Hence,

$$
\begin{aligned}
\left\|x_{n}-u^{\dagger}\right\| & \leq\left[1-(1-k) \frac{\alpha_{n}}{1-\gamma_{n}}\right]\left\|x_{n-1}-u^{\dagger}\right\|+\frac{\alpha_{n}}{1-\gamma_{n}}\left\|f\left(u^{\dagger}\right)-u^{\dagger}\right\|+\frac{\beta_{n}}{1-\gamma_{n}}\left\|V u^{\dagger}-u^{\dagger}\right\| \\
& \leq\left[1-(1-k) \frac{\alpha_{n}}{1-\gamma_{n}}\right]\left\|x_{n-1}-u^{\dagger}\right\|+\frac{\alpha_{n}}{1-\gamma_{n}}\left\|f\left(u^{\dagger}\right)-u^{\dagger}\right\|+\frac{\alpha_{n}}{1-\gamma_{n}}\left\|V u^{\dagger}-u^{\dagger}\right\| \\
& =\left[1-(1-k) \frac{\alpha_{n}}{1-\gamma_{n}}\right]\left\|x_{n-1}-u^{\dagger}\right\|+\frac{\alpha_{n}}{1-\gamma_{n}}\left(\left\|f\left(u^{\dagger}\right)-u^{\dagger}\right\|+\left\|V u^{\dagger}-u^{\dagger}\right\|\right) \\
& \leq \max \left\{\left\|x_{n-1}-u^{\dagger}\right\|,\left(\left\|f\left(u^{\dagger}\right)-u^{\dagger}\right\|+\left\|V u^{\dagger}-u^{\dagger}\right\|\right) /(1-k)\right\} .
\end{aligned}
$$

Thus, $\left\{x_{n}\right\},\left\{T x_{n}\right\},\left\{S x_{n}\right\},\left\{y_{n}\right\},\left\{Q x_{n}\right\}$ and $\left\{A x_{n}\right\}$ are all bounded.

Set $q=J_{\varsigma_{2}}^{M_{2}}\left(u^{\dagger}-\varsigma_{2} A_{2} u^{\dagger}\right)$ and $z_{n}=J_{\varsigma_{1}}^{M_{1}}\left(y_{n}-\varsigma_{1} A_{1} y_{n}\right)$. Then $z_{n}=Q x_{n}, \forall n \geq 1$. By virtue of Lemma 4 , we get

$$
\begin{aligned}
\left\|y_{n}-q\right\|^{2} & =\left\|J_{\varsigma_{2}}^{M_{2}}\left(x_{n}-\varsigma_{2} A_{2} x_{n}\right)-J_{\varsigma_{2}}^{M_{2}}\left(u^{\dagger}-\varsigma_{2} A_{2} u^{\dagger}\right)\right\|^{2} \\
& \leq\left\|x_{n}-u^{\dagger}-\varsigma_{2}\left(A_{2} x_{n}-A_{2} u^{\dagger}\right)\right\|^{2} \\
& \leq\left\|x_{n}-u^{\dagger}\right\|^{2}-2 \varsigma_{2}\left(\zeta_{2}-c^{2} \varsigma_{2}\right)\left\|A_{2} x_{n}-A_{2} u^{\dagger}\right\|^{2},
\end{aligned}
$$

and

$$
\begin{aligned}
\left\|z_{n}-u^{\dagger}\right\|^{2} & =\left\|J_{\varsigma_{1}}^{M_{1}}\left(y_{n}-\varsigma_{1} A_{1} y_{n}\right)-J_{\varsigma_{1}}^{M_{1}}\left(q-\varsigma_{1} A_{1} q\right)\right\|^{2} \\
& \leq\left\|y_{n}-q-\varsigma_{1}\left(A_{1} y_{n}-A_{1} q\right)\right\|^{2} \\
& \leq\left\|y_{n}-q\right\|^{2}-2 \varsigma_{1}\left(\zeta_{1}-c^{2} \varsigma_{1}\right)\left\|A_{1} y_{n}-A_{1} q\right\|^{2} .
\end{aligned}
$$

Substituting (7) for (8), we derive

$$
\left\|z_{n}-u^{\dagger}\right\|^{2} \leq\left\|x_{n}-u^{\dagger}\right\|^{2}-2 \varsigma_{2}\left(\zeta_{2}-c^{2} \varsigma_{2}\right)\left\|A_{2} x_{n}-A_{2} u^{\dagger}\right\|^{2}-2 \varsigma_{1}\left(\zeta_{1}-c^{2} \varsigma_{1}\right)\left\|A_{1} y_{n}-A_{1} q\right\|^{2} .
$$


In view of (5) and (9), we obtain

$$
\begin{aligned}
\left\|x_{n}-u^{\dagger}\right\|^{2}= & \alpha_{n}\left\langle f\left(x_{n-1}\right)-u^{\dagger}, j\left(x_{n}-u^{\dagger}\right)\right\rangle+\delta_{n}\left\langle x_{n-1}-u^{\dagger}, j\left(x_{n}-u^{\dagger}\right)\right\rangle \\
& +\beta_{n}\left\langle V x_{n-1}-u^{\dagger}, j\left(x_{n}-u^{\dagger}\right)\right\rangle+\gamma_{n}\left\langle(1-\mu) S x_{n}+\mu z_{n}-u^{\dagger}, j\left(x_{n}-u^{\dagger}\right)\right\rangle \\
\leq & \alpha_{n}\left\langle f\left(x_{n-1}\right)-u^{\dagger}, j\left(x_{n}-u^{\dagger}\right)\right\rangle+\delta_{n}\left\|x_{n-1}-u^{\dagger}\right\|\left\|x_{n}-u^{\dagger}\right\| \\
& +\beta_{n}\left\|V x_{n-1}-u^{\dagger}\right\|\left\|x_{n}-u^{\dagger}\right\|+\gamma_{n}\left\|(1-\mu) S x_{n}+\mu z_{n}-u^{\dagger}\right\|\left\|x_{n}-u^{\dagger}\right\| \\
\leq & \delta_{n}\left\|x_{n-1}-u^{\dagger}\right\|\left\|x_{n}-u^{\dagger}\right\|+\gamma_{n}\left[(1-\mu)\left\|x_{n}-u^{\dagger}\right\|+\mu\left\|z_{n}-u^{\dagger}\right\|\right]\left\|x_{n}-u^{\dagger}\right\| \\
& +\alpha_{n}\left[\left\langle f\left(x_{n-1}\right)-f\left(u^{\dagger}\right), j\left(x_{n}-u^{\dagger}\right)\right\rangle+\left\langle f\left(u^{\dagger}\right)-u^{\dagger}, j\left(x_{n}-u^{\dagger}\right)\right\rangle\right] \\
& +\beta_{n}\left(\left\|V x_{n-1}-V u^{\dagger}\right\|+\left\|V u^{\dagger}-u^{\dagger}\right\|\right)\left\|x_{n}-u^{\dagger}\right\| \\
\leq & \delta_{n}\left\|x_{n-1}-u^{\dagger}\right\|\left\|x_{n}-u^{\dagger}\right\|+\gamma_{n}\left[(1-\mu)\left\|x_{n}-u^{\dagger}\right\|+\mu\left\|z_{n}-u^{\dagger}\right\|\right]\left\|x_{n}-u^{\dagger}\right\| \\
& +\alpha_{n}\left[k\left\|x_{n-1}-u^{\dagger}\right\|\left\|x_{n}-u^{\dagger}\right\|+\left\langle f\left(u^{\dagger}\right)-u^{\dagger}, j\left(x_{n}-u^{\dagger}\right)\right\rangle\right] \\
& +\beta_{n}\left(\left\|x_{n-1}-u^{\dagger}\right\|+\left\|V u^{\dagger}-u^{\dagger}\right\|\right)\left\|x_{n}-u^{\dagger}\right\| \\
\leq & \alpha_{n}\left\langle f\left(u^{\dagger}\right)-u^{\dagger}, j\left(x_{n}-u^{\dagger}\right)\right\rangle+\left[1-(1-k) \alpha_{n}-\gamma_{n}\right] / 2\left(\left\|x_{n-1}-u^{\dagger}\right\|^{2}+\left\|x_{n}-u^{\dagger}\right\|^{2}\right) \\
& +\beta_{n}\left\|V u^{\dagger}-u^{\dagger}\right\|\left\|x_{n}-u^{\dagger}\right\|+\gamma_{n}\left\|x_{n}-u^{\dagger}\right\|^{2}-\gamma_{n} \mu\left[s_{2}\left(\zeta_{2}-c^{2} \varsigma_{2}\right)\left\|A_{2} x_{n}-A_{2} u^{\dagger}\right\|^{2}\right. \\
& \left.+\varsigma_{1}\left(\zeta_{1}-c^{2} \varsigma_{1}\right)\left\|A_{1} y_{n}-A_{1} q\right\|^{2}\right] .
\end{aligned}
$$

\section{It follows that}

$$
\begin{aligned}
& \gamma_{n} \mu\left[\zeta_{2}\left(\zeta_{2}-c^{2} \varsigma_{2}\right)\left\|A_{2} x_{n}-A_{2} u^{\dagger}\right\|^{2}+\varsigma_{1}\left(\zeta_{1}-c^{2} \zeta_{1}\right)\left\|A_{1} y_{n}-A_{1} q\right\|^{2}\right] \\
& \leq {\left[1-(1-k) \alpha_{n}-\gamma_{n}\right] / 2\left(\left\|x_{n-1}-u^{\dagger}\right\|^{2}+\left\|x_{n}-u^{\dagger}\right\|^{2}\right)+\alpha_{n}\left\langle f\left(u^{\dagger}\right)-u^{\dagger}, j\left(x_{n}-u^{\dagger}\right)\right\rangle } \\
&+\beta_{n}\left\|V u^{\dagger}-u^{\dagger}\right\|\left\|x_{n}-u^{\dagger}\right\|-\left(1-\gamma_{n}\right)\left\|x_{n}-u^{\dagger}\right\|^{2} \\
& \leq \alpha_{n}\left\|f\left(u^{\dagger}\right)-u^{\dagger}\right\|\left\|x_{n}-u^{\dagger}\right\|+\left[1-(1-k) \alpha_{n}-\gamma_{n}\right] / 2\left(\left\|x_{n-1}-u^{\dagger}\right\|^{2}+\left\|x_{n}-u^{\dagger}\right\|^{2}\right) \\
&+\beta_{n}\left\|V u^{\dagger}-u^{\dagger}\right\|\left\|x_{n}-u^{\dagger}\right\| .
\end{aligned}
$$

By the assumptions (ii) and (iii), we conclude

$$
\lim _{n \rightarrow \infty}\left\|A_{2} x_{n}-A_{2} u^{\dagger}\right\|=0 \text { and } \lim _{n \rightarrow \infty}\left\|A_{1} y_{n}-A_{1} q\right\|=0 .
$$

Utilizing Lemma 1 and Proposition 1, we have

$$
\begin{aligned}
\left\|y_{n}-q\right\|^{2} & =\left\|J_{\varsigma_{2}}^{M_{2}}\left(x_{n}-\varsigma_{2} A_{2} x_{n}\right)-J_{\varsigma_{2}}^{M_{2}}\left(u^{\dagger}-\varsigma_{2} A_{2} u^{\dagger}\right)\right\|^{2} \\
& \leq\left\langle\left(x_{n}-\varsigma_{2} A_{2} x_{n}\right)-\left(u^{\dagger}-\varsigma_{2} A_{2} u^{\dagger}\right), j\left(y_{n}-q\right)\right\rangle \\
& =\left\langle x_{n}-u^{\dagger}, j\left(y_{n}-q\right)\right\rangle+\varsigma_{2}\left\langle A_{2} u^{\dagger}-A_{2} x_{n}, j\left(y_{n}-q\right)\right\rangle \\
& \leq\left[\left\|x_{n}-u^{\dagger}\right\|^{2}+\left\|y_{n}-u^{\dagger}\right\|^{2}-g_{1}\left(\left\|x_{n}-y_{n}-\left(u^{\dagger}-q\right)\right\|\right)\right] / 2+\varsigma_{2}\left\|A_{2} u^{\dagger}-A_{2} x_{n}\right\|\left\|y_{n}-q\right\| .
\end{aligned}
$$

It follows that

$$
\left\|y_{n}-q\right\|^{2} \leq\left\|x_{n}-u^{\dagger}\right\|^{2}-g_{1}\left(\left\|x_{n}-y_{n}-\left(u^{\dagger}-q\right)\right\|\right)+2 \varsigma_{2}\left\|A_{2} u^{\dagger}-A_{2} x_{n}\right\|\left\|y_{n}-q\right\| .
$$

Similarly,

$$
\begin{aligned}
\left\|z_{n}-u^{\dagger}\right\|^{2} & =\left\|J_{\zeta_{1}}^{M_{1}}\left(y_{n}-\varsigma_{1} A_{1} y_{n}\right)-J_{\zeta_{1}}^{M_{1}}\left(q-\varsigma_{1} A_{1} q\right)\right\|^{2} \\
& \leq\left\langle\left(y_{n}-\varsigma_{1} A_{1} y_{n}\right)-\left(q-\varsigma_{1} A_{1} q\right), j\left(z_{n}-u^{\dagger}\right)\right\rangle \\
& =\left\langle y_{n}-q, j\left(z_{n}-q\right)\right\rangle+\varsigma_{1}\left\langle A_{1} q-A_{1} y_{n}, j\left(z_{n}-u^{\dagger}\right)\right\rangle \\
& \leq \frac{1}{2}\left[\left\|y_{n}-q\right\|^{2}+\left\|z_{n}-u^{\dagger}\right\|^{2}-g_{2}\left(\left\|y_{n}-z_{n}+\left(u^{\dagger}-q\right)\right\|\right)\right]+\varsigma_{1}\left\|A_{1} q-A_{1} y_{n}\right\|\left\|z_{n}-u^{\dagger}\right\|,
\end{aligned}
$$


which implies that

$$
\left\|z_{n}-u^{\dagger}\right\|^{2} \leq\left\|y_{n}-q\right\|^{2}-g_{2}\left(\left\|y_{n}-z_{n}+\left(u^{\dagger}-q\right)\right\|\right)+2 \varsigma_{1}\left\|A_{1} q-A_{1} y_{n}\right\|\left\|z_{n}-u^{\dagger}\right\| .
$$

Substituting (12) for (13), we get

$$
\begin{aligned}
\left\|z_{n}-u^{\dagger}\right\|^{2} \leq & \left\|x_{n}-u^{\dagger}\right\|^{2}-g_{1}\left(\left\|x_{n}-y_{n}-\left(u^{\dagger}-q\right)\right\|\right)-g_{2}\left(\left\|y_{n}-z_{n}+\left(u^{\dagger}-q\right)\right\|\right) \\
& +2 \varsigma_{2}\left\|A_{2} u^{\dagger}-A_{2} x_{n}\right\|\left\|y_{n}-q\right\|+2 \varsigma_{1}\left\|A_{1} q-A_{1} y_{n}\right\|\left\|z_{n}-u^{\dagger}\right\| .
\end{aligned}
$$

From (10) and (14), we have

$$
\begin{aligned}
\left\|x_{n}-u^{\dagger}\right\|^{2} \leq & \alpha_{n}\left\langle f\left(u^{\dagger}\right)-u^{\dagger}, j\left(x_{n}-u^{\dagger}\right)\right\rangle+\left[1-(1-k) \alpha_{n}-\gamma_{n}\right]\left(\left\|x_{n-1}-u^{\dagger}\right\|^{2}+\left\|x_{n}-u^{\dagger}\right\|^{2}\right) / 2 \\
& +\beta_{n}\left\|V u^{\dagger}-u^{\dagger}\right\|\left\|x_{n}-u^{\dagger}\right\|+\gamma_{n}\left(\left\|x_{n}-u^{\dagger}\right\|^{2}+(1-\mu)\left\|x_{n}-u^{\dagger}\right\|^{2}+\mu\left\|z_{n}-u^{\dagger}\right\|^{2}\right) / 2 \\
\leq & \alpha_{n}\left\langle f\left(u^{\dagger}\right)-u^{\dagger}, j\left(x_{n}-u^{\dagger}\right)\right\rangle+\left[1-(1-k) \alpha_{n}-\gamma_{n}\right]\left(\left\|x_{n-1}-u^{\dagger}\right\|^{2}+\left\|x_{n}-u^{\dagger}\right\|^{2}\right) / 2 \\
& +\beta_{n}\left\|V u^{\dagger}-u^{\dagger}\right\|\left\|x_{n}-u^{\dagger}\right\|+\gamma_{n}\left\|x_{n}-u^{\dagger}\right\|^{2}-\frac{\gamma_{n} \mu}{2}\left[g_{1}\left(\left\|x_{n}-y_{n}-\left(u^{\dagger}-q\right)\right\|\right)\right. \\
& \left.+g_{2}\left(\left\|y_{n}-z_{n}+\left(u^{\dagger}-q\right)\right\|\right)\right]+\gamma_{n} \mu\left(\varsigma_{2}\left\|A_{2} u^{\dagger}-A_{2} x_{n}\right\|\left\|y_{n}-q\right\|\right. \\
& \left.+\varsigma_{1}\left\|A_{1} q-A_{1} y_{n}\right\|\left\|z_{n}-u^{\dagger}\right\|\right) .
\end{aligned}
$$

It follows that

$$
\begin{aligned}
& \frac{\gamma_{n} \mu}{2}\left[g_{1}\left(\left\|x_{n}-y_{n}-\left(u^{\dagger}-q\right)\right\|\right)+g_{2}\left(\left\|y_{n}-z_{n}+\left(u^{\dagger}-q\right)\right\|\right)\right] \\
& \leq \alpha_{n}\left\langle f\left(u^{\dagger}\right)-u^{\dagger}, j\left(x_{n}-u^{\dagger}\right)\right\rangle+\left[1-(1-k) \alpha_{n}-\gamma_{n}\right]\left(\left\|x_{n-1}-u^{\dagger}\right\|^{2}+\left\|x_{n}-u^{\dagger}\right\|^{2}\right) / 2 \\
& \quad+\beta_{n}\left\|V u^{\dagger}-u^{\dagger}\right\|\left\|x_{n}-u^{\dagger}\right\|-\left(1-\gamma_{n}\right)\left\|x_{n}-u^{\dagger}\right\|^{2}+\gamma_{n} \mu\left(\varsigma_{2}\left\|A_{2} u^{\dagger}-A_{2} x_{n}\right\|\left\|y_{n}-q\right\|\right. \\
& \left.\quad+\varsigma_{1}\left\|A_{1} q-A_{1} y_{n}\right\|\left\|z_{n}-u^{\dagger}\right\|\right) \\
& \leq \alpha_{n}\left\|f\left(u^{\dagger}\right)-u^{\dagger}\right\|\left\|x_{n}-u^{\dagger}\right\|+\left[1-(1-k) \alpha_{n}-\gamma_{n}\right]\left(\left\|x_{n-1}-u^{\dagger}\right\|^{2}+\left\|x_{n}-u^{\dagger}\right\|^{2}\right) / 2 \\
& \quad+\beta_{n}\left\|V u^{\dagger}-u^{\dagger}\right\|\left\|x_{n}-u^{\dagger}\right\|+\varsigma_{2}\left\|A_{2} u^{\dagger}-A_{2} x_{n}\right\|\left\|y_{n}-q\right\|+\varsigma_{1}\left\|A_{1} q-A_{1} y_{n}\right\|\left\|z_{n}-u^{\dagger}\right\| .
\end{aligned}
$$

This together with conditions (ii) and (iii) implies that

$$
\lim _{n \rightarrow \infty} g_{1}\left(\left\|x_{n}-y_{n}-\left(u^{\dagger}-q\right)\right\|\right)=0 \text { and } \lim _{n \rightarrow \infty} g_{2}\left(\left\|y_{n}-z_{n}+\left(u^{\dagger}-q\right)\right\|\right)=0 .
$$

Hence,

$$
\lim _{n \rightarrow \infty}\left\|x_{n}-y_{n}-\left(u^{\dagger}-q\right)\right\|=0 \text { and } \lim _{n \rightarrow \infty}\left\|y_{n}-z_{n}+\left(u^{\dagger}-q\right)\right\|=0 .
$$

In light of (15), we have

$$
\left\|x_{n}-z_{n}\right\| \leq\left\|x_{n}-y_{n}-\left(u^{\dagger}-q\right)\right\|+\left\|y_{n}-z_{n}+\left(u^{\dagger}-q\right)\right\| \rightarrow 0,
$$

which means that

$$
\lim _{n \rightarrow \infty}\left\|x_{n}-Q x_{n}\right\|=0
$$

Note that

$$
\begin{aligned}
\gamma_{n}\left\|x_{n}-A x_{n}\right\| & =\left\|\alpha_{n}\left(f\left(x_{n-1}\right)-x_{n}\right)+\delta_{n}\left(x_{n-1}-x_{n}\right)+\beta_{n}\left(V x_{n-1}-x_{n}\right)\right\| \\
& \leq \alpha_{n}\left\|f\left(x_{n-1}\right)-x_{n}\right\|+\delta_{n}\left\|x_{n-1}-x_{n}\right\|+\beta_{n}\left\|V x_{n-1}-x_{n}\right\| .
\end{aligned}
$$

Thus,

$$
\lim _{n \rightarrow \infty}\left\|x_{n}-A x_{n}\right\|=0 .
$$


Also, observe that

$$
\mu\left\|S x_{n}-x_{n}\right\|=\left\|A x_{n}-x_{n}-(1-\mu)\left(Q x_{n}-x_{n}\right)\right\| \leq\left\|A x_{n}-x_{n}\right\|+\left\|Q x_{n}-x_{n}\right\| .
$$

In terms of (16) and (17), we obtain

$$
\lim _{n \rightarrow \infty}\left\|x_{n}-S x_{n}\right\|=0
$$

Since $S=(1-\alpha) I+\alpha T$ with $0<\alpha<\min \left\{1, \frac{\lambda}{c^{2}}\right\}$, it is easy from (3.15) that

$$
\lim _{n \rightarrow \infty}\left\|x_{n}-T x_{n}\right\|=0 .
$$

Define a net $\left\{u_{t}\right\}$ by $u_{t}=(1-t) A u_{t}+t f\left(u_{t}\right)$. So,

$$
u_{t}-x_{n}=t\left(f\left(u_{t}\right)-x_{n}\right)+(1-t)\left(A u_{t}-x_{n}\right) .
$$

It follows that

$$
\begin{aligned}
\left\|u_{t}-x_{n}\right\|^{2} \leq & 2 t\left\langle f\left(u_{t}\right)-x_{n}, j\left(u_{t}-x_{n}\right)\right\rangle+(1-t)^{2}\left\|A u_{t}-x_{n}\right\|^{2} \\
\leq & 2 t\left\langle f\left(u_{t}\right)-x_{n}, j\left(u_{t}-x_{n}\right)\right\rangle+(1-t)^{2}\left[\left\|A u_{t}-A x_{n}\right\|+\left\|A x_{n}-x_{n}\right\|\right]^{2} \\
\leq & 2 t\left\langle f\left(u_{t}\right)-x_{n}, j\left(u_{t}-x_{n}\right)\right\rangle+(1-t)^{2}\left[\left\|u_{t}-x_{n}\right\|+\left\|A x_{n}-x_{n}\right\|\right]^{2} \\
= & (1-t)^{2}\left[\left\|u_{t}-x_{n}\right\|^{2}+\left\|A x_{n}-x_{n}\right\|^{2}+2\left\|u_{t}-x_{n}\right\|\left\|A x_{n}-x_{n}\right\|\right] \\
& +2 t\left\langle f\left(u_{t}\right)-x_{n}, j\left(u_{t}-x_{n}\right)\right\rangle,
\end{aligned}
$$

that is,

$$
\begin{aligned}
\left\|u_{t}-x_{n}\right\|^{2} \leq & \left\|A x_{n}-x_{n}\right\|\left(2\left\|u_{t}-x_{n}\right\|+\left\|A x_{n}-x_{n}\right\|\right)+2 t\left\|u_{t}-x_{n}\right\|^{2} \\
& +2 t\left\langle f\left(u_{t}\right)-u_{t}, j\left(u_{t}-x_{n}\right)\right\rangle+(1-t)^{2}\left\|u_{t}-x_{n}\right\|^{2} \\
= & \left(1+t^{2}\right)\left\|u_{t}-x_{n}\right\|^{2}+2 t\left\langle f\left(u_{t}\right)-u_{t}, j\left(u_{t}-x_{n}\right)\right\rangle \\
& +\left\|A x_{n}-x_{n}\right\|\left(2\left\|u_{t}-x_{n}\right\|+\left\|A x_{n}-x_{n}\right\|\right) .
\end{aligned}
$$

It follows that

$$
\left\langle u_{t}-f\left(u_{t}\right), j\left(u_{t}-x_{n}\right)\right\rangle \leq \frac{t}{2}\left\|u_{t}-x_{n}\right\|^{2}+\frac{1}{2 t}\left(2\left\|u_{t}-x_{n}\right\|+\left\|A x_{n}-x_{n}\right\|\right)\left\|A x_{n}-x_{n}\right\| .
$$

Letting $n \rightarrow \infty$ in (20), from (17), we have

$$
\varlimsup_{n \rightarrow \infty}\left\langle u_{t}-f\left(u_{t}\right), j\left(u_{t}-x_{n}\right)\right\rangle \leq \frac{t M_{0}}{2}
$$

where $M_{0}$ is a constant such that $\left\|u_{t}-x_{n}\right\|^{2} \leq M_{0}, \forall n \geq 0, \forall t \in(0,1)$. By Lemma $6, u_{t} \rightarrow x^{*} \in \Omega$, which solves $\left\langle(I-f) x^{*}, j\left(x^{*}-x\right)\right\rangle \leq 0, \forall x \in \Omega$. Letting $t \rightarrow 0^{+}$in (21), we deduce

$$
\varlimsup_{n \rightarrow \infty}\left\langle x^{*}-f\left(x^{*}\right), j\left(x^{*}-x_{n}\right)\right\rangle \leq 0 .
$$

Putting $u^{+}=x^{*}$ in (10), we obtain

$$
\begin{aligned}
\left\|x_{n}-x^{*}\right\|^{2} \leq & \alpha_{n}\left\langle f\left(x^{*}\right)-x^{*}, j\left(x_{n}-x^{*}\right)\right\rangle+\left[1-(1-k) \alpha_{n}-\gamma_{n}\right]\left(\left\|x_{n-1}-x^{*}\right\|^{2}+\left\|x_{n}-x^{*}\right\|^{2}\right) / 2 \\
& +\beta_{n}\left\|V x^{*}-x^{*}\right\|\left\|x_{n}-x^{*}\right\|+\gamma_{n}\left\|x_{n}-x^{*}\right\|^{2} .
\end{aligned}
$$


Consequently, we have

$$
\begin{aligned}
\left\|x_{n}-x^{*}\right\|^{2} \leq & \frac{2 \alpha_{n}}{1+(1-k) \alpha_{n}-\gamma_{n}}\left\langle f\left(x^{*}\right)-x^{*}, j\left(x_{n}-x^{*}\right)\right\rangle+\frac{1-(1-k) \alpha_{n}-\gamma_{n}}{1+(1-k) \alpha_{n}-\gamma_{n}}\left\|x_{n-1}-x^{*}\right\|^{2} \\
& +\frac{2 \beta_{n}}{1+(1-k) \alpha_{n}-\gamma_{n}}\left\|V x^{*}-x^{*}\right\|\left\|x_{n}-x^{*}\right\| \\
= & \left(1-v_{n}\right)\left\|x_{n-1}-x^{*}\right\|^{2}+v_{n} \varrho_{n},
\end{aligned}
$$

where $v_{n}=\frac{2(1-k) \alpha_{n}}{1+(1-k) \alpha_{n}-\gamma_{n}}$ and

$$
\varrho_{n}=\frac{\beta_{n}}{(1-k) \alpha_{n}}\left\|V x^{*}-x^{*}\right\|\left\|x_{n}-x^{*}\right\|+\frac{1}{1-k}\left\langle f\left(x^{*}\right)-x^{*}, j\left(x_{n}-x^{*}\right)\right\rangle .
$$

Now, observe that

$$
(1-k) \alpha_{n}=\frac{2(1-k) \alpha_{n}}{2} \leq \frac{2(1-k) \alpha_{n}}{1-\gamma_{n}+(1-k) \alpha_{n}}=v_{n} .
$$

Observe that $\varlimsup_{n \rightarrow \infty} \varrho_{n} \leq 0$. With the help of Lemma 7, we get $x_{n} \rightarrow x^{*}$. Moreover, putting $u^{\dagger}=x^{*}$ and $q=y^{*}=J_{\varsigma_{2}}^{M_{2}}\left(I-\varsigma_{2} A_{2}\right) x^{*}$ in (15), we obtain

$$
\lim _{n \rightarrow \infty}\left\|x_{n}-y_{n}-\left(x^{*}-y^{*}\right)\right\|=0 .
$$

Note that

$$
\left\|y_{n}-y^{*}\right\| \leq\left\|x_{n}-y_{n}-\left(x^{*}-y^{*}\right)\right\|+\left\|x^{*}-x_{n}\right\| .
$$

So, it follows that $y_{n} \rightarrow y^{*}$ as $n \rightarrow \infty$. Consequently, $\left(x^{*}, y^{*}\right)$ is a solution of (1) by Lemma 2 .

Corollary 1. Let $X$ be a uniformly convex and 2-uniformly smooth Banach space and $\varnothing \neq C \subset X a$ closed convex set. Let $M: C \rightarrow 2^{X}$ be an m-accretive operator and $A: C \rightarrow X$ be a $\zeta$-inverse-strongly accretive operator. Let $f: C \rightarrow C$ be a contraction with coefficient $k \in[0,1)$. Let $V: C \rightarrow C$ be a nonexpansive operator and $T: C \rightarrow C$ be a $\lambda$-strict pseudocontraction with $\Omega:=F(T) \cap F(Q) \neq \varnothing$, where the operator $Q=J_{\zeta_{1}}^{M}\left(I-\varsigma_{1} A\right) J_{\varsigma_{2}}^{M}\left(I-\varsigma_{2} A\right)$ and $0<\varsigma_{i}<\frac{\zeta}{c^{2}}(i=1,2)$. Assume that the sequences $\left\{\alpha_{n}\right\} \subset(0,1),\left\{\beta_{n}\right\} \subset(0,1)$ and $\left\{\gamma_{n}\right\} \subset(0,1)$ satisfy

(i) $\alpha_{n}+\delta_{n}+\beta_{n}+\gamma_{n}=1(\forall n \geq 1)$;

(ii) $\alpha_{n} \rightarrow 0$ and $\frac{\beta_{n}}{\alpha_{n}} \rightarrow 0$;

(iii) $\gamma_{n} \rightarrow 1$;

(iv) $\sum_{n=1}^{\infty} \alpha_{n}=\infty$.

Given $x_{0} \in C$, compute the sequences $\left\{x_{n}\right\}$ and $\left\{y_{n}\right\}$ such that

$$
\left\{\begin{array}{l}
y_{n}=J_{\zeta_{2}}^{M}\left(x_{n}-\varsigma_{2} A x_{n}\right), \\
x_{n}=\delta_{n} x_{n-1}+\alpha_{n} f\left(x_{n-1}\right)+\beta_{n} V x_{n-1}+\gamma_{n}\left[\mu S x_{n}+(1-\mu) J_{\zeta_{1}}^{M}\left(y_{n}-\varsigma_{1} A y_{n}\right)\right], \forall n \geq 1,
\end{array}\right.
$$

where $S x=(1-\alpha) x+\alpha T x, \forall x \in C$ with $0<\alpha<\min \left\{1, \frac{\lambda}{c^{2}}\right\}$ and $\mu \in(0,1)$. Then $x_{n} \rightarrow x^{*}, y_{n} \rightarrow y^{*}$ and

(a) $\left(x^{*}, y^{*}\right)$ solves the GSVI (2);

(b) $x^{*}$ solves the variational inequality: $\left\langle(I-f) x^{*}, j\left(u-x^{*}\right)\right\rangle \geq 0, \forall u \in \Omega$.

Corollary 2. Let $H$ be a Hilbert space and $\varnothing \neq C \subset H$ a closed convex set. Let $M: C \rightarrow 2^{H}$ be a maximal monotone operator and $A: C \rightarrow H$ be a $\zeta$-inverse-strongly monotone operator. Let $f: C \rightarrow C$ be a contraction 
with coefficient $k \in[0,1)$. Let $V: C \rightarrow C$ be a nonexpansive operator and $T: C \rightarrow C$ be a $\lambda$-strict pseudocontraction with $\Omega:=F(T) \cap F(Q) \neq \varnothing$, where the operator $Q=J_{\zeta_{1}}^{M}\left(I-\varsigma_{1} A\right) J_{\varsigma_{2}}^{M}\left(I-\varsigma_{2} A\right)$ and $0<\varsigma_{i}<\frac{\zeta}{c^{2}}(i=1,2)$. Assume that the sequences $\left\{\alpha_{n}\right\} \subset(0,1),\left\{\beta_{n}\right\} \subset(0,1)$ and $\left\{\gamma_{n}\right\} \subset(0,1)$ satisfy

(i) $\alpha_{n}+\delta_{n}+\beta_{n}+\gamma_{n}=1(\forall n \geq 1)$;

(ii) $\alpha_{n} \rightarrow 0$ and $\frac{\beta_{n}}{\alpha_{n}} \rightarrow 0$;

(iii) $\gamma_{n} \rightarrow 1$;

(iv) $\sum_{n=1}^{\infty} \alpha_{n}=\infty$.

Given $x_{0} \in C$, compute the sequences $\left\{x_{n}\right\}$ and $\left\{y_{n}\right\}$ such that

$$
\left\{\begin{array}{l}
y_{n}=J_{\zeta_{2}}^{M}\left(x_{n}-\varsigma_{2} A x_{n}\right), \\
x_{n}=\delta_{n} x_{n-1}+\alpha_{n} f\left(x_{n-1}\right)+\beta_{n} V x_{n-1}+\gamma_{n}\left[\mu S x_{n}+(1-\mu) J_{\zeta_{1}}^{M}\left(y_{n}-\varsigma_{1} A y_{n}\right)\right], \forall n \geq 1,
\end{array}\right.
$$

where $S x=(1-\alpha) x+\alpha T x, \forall x \in C$ with $0<\alpha<\min \left\{1, \frac{\lambda}{c^{2}}\right\}$ and $\mu \in(0,1)$. Then $x_{n} \rightarrow x^{*}, y_{n} \rightarrow y^{*}$ and

(a) $\left(x^{*}, y^{*}\right)$ solves the GSVI (2);

(b) $x^{*}$ solves the variational inequality: $\left\langle(I-f) x^{*}, j\left(u-x^{*}\right)\right\rangle \geq 0, \forall u \in \Omega$.

\section{Conclusions}

In this paper, we consider the GSVI (1) with the hierarchical variational inequality (HVI) constraint for a strict pseudocontraction in a uniformly convex and 2-uniformly smooth Banach space. By utilizing the equivalence between the GSVI (1) and the fixed point problem, we construct an implicit composite viscosity approximation method for solving the GSVI (1) with the HVI constraint for strict pseudocontractions. We prove the strong convergence of the proposed algorithm to a solution of the GSVI (1) with the HVI constraint for strict pseudocontraction under very mild conditions. Note that our algorithm (4) is an implicit manner. This brings us a natural question: could we construct an explicit algorithm with strong convergence?

Author Contributions: All the authors have contributed equally to this paper. All the authors have read and approved the final manuscript.

Funding: This research was partially supported by the Innovation Program of Shanghai Municipal Education Commission (15ZZ068), Ph.D. Program Foundation of Ministry of Education of China (20123127110002) and Program for Outstanding Academic Leaders in Shanghai City (15XD1503100). Yonghong Yao was supported in part by the grant TD13-5033.

Conflicts of Interest: The authors declare no conflict of interest.

\section{References}

1. Lions, J.L.; Stampacchia, G. Variational inequalities. Commun. Pure Appl. Math. 1967, 20, 493-517. [CrossRef]

2. Ceng, L.C.; Guu, S.M.; Yao, J.C. Weak convergence theorem by a modified extragradient method for variational inclusions, variational inequalities and fixed point problems. J. Nonlinear Convex Anal. 2013, 14, 21-31.

3. Ceng, L.C.; Latif, A.; Yao, J.C. On solutions of a system of variational inequalities and fixed point problems in Banach spaces. Fixed Point Theory Appl. 2013, 2013, 176. [CrossRef]

4. Ceng, L.C.; Wang, C.Y.; Yao, J.C. Strong convergence theorems by a relaxed extragradient method for a general system of variational inequalities. Math. Methods Oper. Res. 2008, 67, 375-390. [CrossRef]

5. Ceng, L.C.; Latif, A.; Ansari, Q.H.; Yao, J.C. Hybrid extragradient method for hierarchical variational inequalities. Fixed Point Theory Appl. 2014, 2014, 222. [CrossRef]

6. Chinedu, I.; Christian, O.C.; Felicia, O.I. A viscosity iterative technique for split variational inclusion and fixed point problems between a Hilbert space and a Banach space. J. Fixed Point Theory Appl. 2018, 20, 157.

7. Yao, Y.; Liou, Y.C.; Kang, S.M. Approach to common elements of variational inequality problems and fixed point problems via a relaxed extragradient method. Comput. Math. Appl. 2010, 59, 3472-3480. [CrossRef] 
8. Ceng, L.C.; Plubtieng, S.; Wong, M.M.; Yao, J.C. System of variational inequalities with constraints of mixed equilibria, variational inequalities, and convex minimization and fixed point problems. J. Nonlinear Convex Anal. 2015, 16, 385-421.

9. Yao, Y.; Postolache, M.; Zhu, Z. Gradient methods with selection technique for the multiple-sets split feasibility problem. Optimization 2019. [CrossRef]

10. Pongsakorn, S.; Prasit, C. Iterative methods for solving quasi-variational inclusion and fixed point problem in q-uniformly smooth Banach spaces. Numer. Algor. 2018, 78, 1019-1044.

11. Buong, N.; Hoai, P.T. Iterative methods for zeros of a monotone variational inclusion in Hilbert spaces. Calcolo 2018, 55, 7. [CrossRef]

12. E Jeremiah Nkwegu, Z.; Chinedu, I. Iterative Approximation of Solution of Split Variational Inclusion Problem. Filomat 2018, 32, 2921-2932.

13. Zhang, C.; Wang, Y. Proximal algorithm for solving monotone variational inclusion. Optimization 2018, 67, 1197-1209. [CrossRef]

14. Ceng, L.C.; Guu, S.M.; Yao, J.C. Hybrid viscosity CQ method for finding a common solution of a variational inequality, a general system of variational inequalities, and a fixed point problem. Fixed Point Theory Appl. 2013, 2013, 313. [CrossRef]

15. Yao, Y.; Chen, R.; Xu, H.K. Schemes for finding minimum-norm solutions of variational inequalities. Nonlinear Anal. 2010, 72, 3447-3456. [CrossRef]

16. Prashanta, M.; Chandal, N. On inertial proximal algorithm for split variational inclusion problems. Optimization 2018, 67, 1701-1716.

17. Zhao, X.P.; Sahu, D.R.; Wen, C.F. Iterative methods for system of variational inclusions involving accretive operators and applications. Fixed Point Theory 2018, 19, 801-822. [CrossRef]

18. Yao, Y.; Postolache, M.; Yao, J.C. An iterative algorithm for solving the generalized variational inequalities and fixed points problems. Mathematics 2019, 1, 61. [CrossRef]

19. He, X.F.; Lou, J.; He, Z. Iterative methods for solving variational inclusions in Banach spaces. J. Comput. Appl. Math. 2007, 203, 80-86. [CrossRef]

20. Chang, S.S.; Wen, C.F.; Yao, J.C. A generalized forward-backward splitting method for solving a system of quasi variational inclusions in Banach spaces. Revista de la Real Academia de Ciencias Exactas Fisicas y Naturales Serie A Matematicas 2019, 113, 729-747. [CrossRef]

21. Zegeye, H.; Shahzad, N.; Yao, Y.H. Minimum-norm solution of variational inequality and fixed point problem in Banach spaces. Optimization 2015, 64, 453-471. [CrossRef]

22. Thakur, B.-S.; Postolache, M. Existence and approximation of solutions for generalized extended nonlinear variational inequalities. J. Inequal. Appl. 2013, 2013, 590. [CrossRef]

23. Dong, Q.-L.; Cho, Y.-J.; Zhong, L.-L.; Rassias, T.-M. Inertial projection and contraction algorithms for variational inequalities. J. Glob. Optim. 2018, 7, 687-704. [CrossRef]

24. Yao, Y.; Liou, Y.C.; Yao, J.C. Iterative algorithms for the split variational inequality and fixed point problems under nonlinear transformations. J. Nonlinear Sci. Appl. 2017, 10, 843-854. [CrossRef]

25. Dong, Q.-L.; Cho, Y.-J.; Rassias, T.-M. The projection and contraction methods for finding common solutions to variational inequality problems. Optim. Lett. 2018, 12, 1871-1896. [CrossRef]

26. Ceng, L.C.; Ansari, Q.H.; Yao, J.C. Relaxed extragradient iterative methods for variational inequalities. Appl. Math. Comput. 2011, 218, 1112-1123. [CrossRef]

27. Yao, Y.H.; Postolache, M.; Liou, Y.C.; Yao, Z.S. Construction algorithms for a class of monotone variational inequalities. Optim. Lett. 2016, 10, 1519-1528. [CrossRef]

28. Lan, H.Y.; Cho, Y.J.; Verma, R.U. Nonlinear relaxed cocoercive variational inclusions involving $(A, \eta)$-accretive mappings in Banach spaces. Comput. Math. Appl. 2006, 51, 1529-1538. [CrossRef]

29. Xiong, T.J.; Lan, H.Y. On general system of generalized quasi-variational-like inclusions with maximal eta-monotone mappings in Hilbert spaces. J. Comput. Anal. Appl. 2015, 18, 506-514.

30. Qin, X.; Chang, S.S.; Cho, Y.J.; Kang, S.M. Approximation of solutions to a system of variational inclusions in Banach spaces. J. Inequal. Appl. 2010, 2010, 916806. [CrossRef]

31. Jung, J.S. Strong convergence of viscosity approximation methods for finding zeros of accretive operators in Banach spaces. Nonlinear Anal. 2010, 72, 449-459. [CrossRef]

32. Yao, Y.H.; Leng, L.; Postolache, M.; Zheng, X. Mann-type iteration method for solving the split common fixed point problem. J. Nonlinear Convex Anal. 2017, 18, 875-882. 
33. Zhou, H. Convergence theorems for $\lambda$-strict pseudo-contractions in 2-uniformly smooth Banach spaces. Nonlinear Anal. 2008, 69, 3160-3173. [CrossRef]

34. Zhao, X.P.; Ng, K.F.; Li, C.; Yao, J.C. Linear regularity and linear convergence of projection-based methods for solving convex feasibility problems. Appl. Math. Optim. 2018, 78, 613-641. [CrossRef]

35. Yao, Y.; Yao, J.C.; Liou, Y.C.; Postolache, M. Iterative algorithms for split common fixed points of demicontractive operators without priori knowledge of operator norms. Carpathian J. Math. 2018, 34, 459-466.

36. Yao, Y.; Liou, Y.C.; Postolache, M. Self-adaptive algorithms for the split problem of the demicontractive operators. Optim. 2018, 67, 1309-1319. [CrossRef]

37. Zhang, S.S.; Lee Joseph, H.W.; Chan, C.K. Algorithms of common solutions for quasi variational inclusion and fixed point problems. Appl. Math. Mechan. 2008, 29, 571-581. [CrossRef]

38. Peng, J.W.; Wang, Y.; Shyu, D.S.; Yao, J.C. Common solutions of an iterative scheme for variational inclusions, equilibrium problems and fixed point problems. J. Inequal. Appl. 2008, 2008, 720371. [CrossRef]

(C) 2019 by the authors. Licensee MDPI, Basel, Switzerland. This article is an open access article distributed under the terms and conditions of the Creative Commons Attribution (CC BY) license (http:/ / creativecommons.org/licenses/by/4.0/). 